it is difficult to know, but it is a problem that calls for consideration, and this might be preferable to awaiting a slower recovery from severe bleeding. There is much to be said in favour of immediate blood transfusion.

\title{
REFERENCES
}

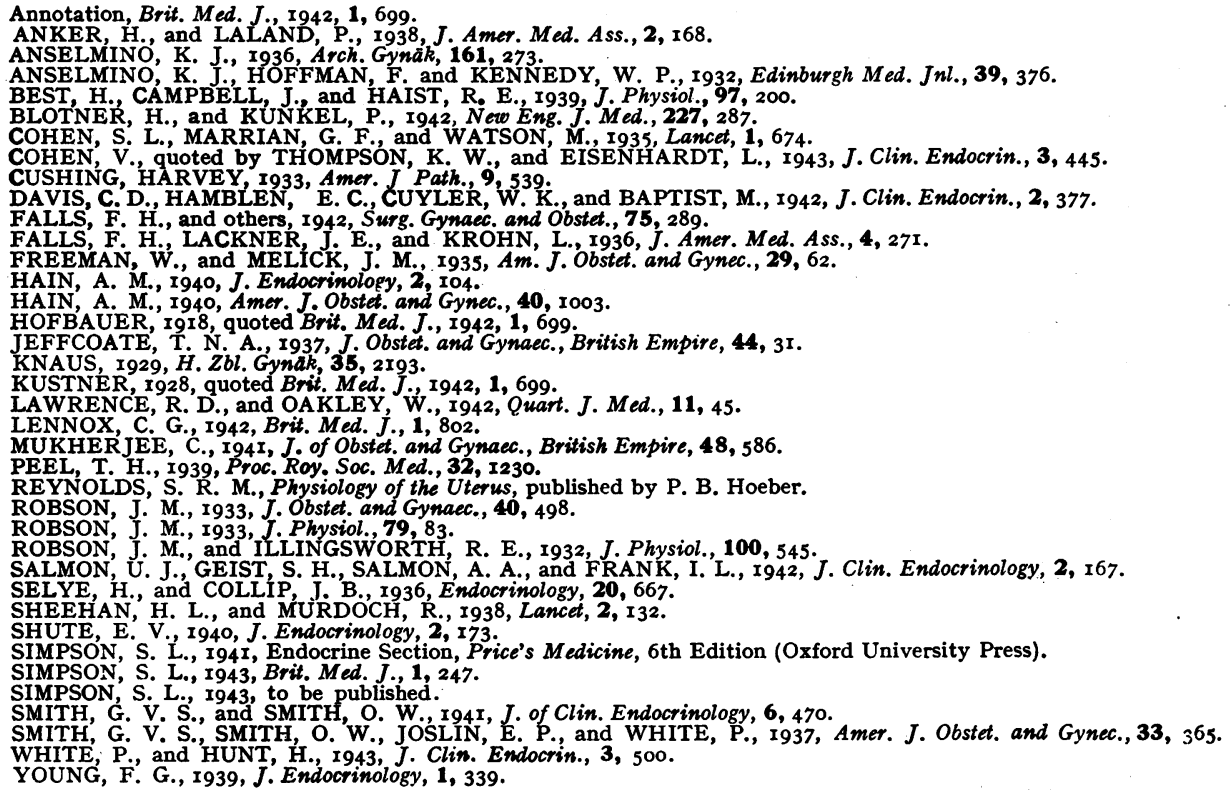

\section{BLOOD DISORDERS IN RELATION TO PREGNANCY}

\author{
By A. PINEY, M.D., M.R.C.P. \\ (Physician, St. Mary's Hospital, E.r3)
}

It is, of course, impossible, in the limits of a single article, to discuss all that is known of blood changes during pregnancy; and only those alterations that are recognised to be of practical significance will be considered. It is, however, timely to emphasise that our knowledge of the blood in normal and abnormal pregnancy is woefully deficient; and a great deal more observation is needed to fill the large gaps in our knowledge.

\section{Physiological changes.}

The development of the foetus, the increase in bulk of the uterus, the placental circulation, and the blood lost during parturition make considerable demands on the maternal stores of iron. Indeed, it has been estimated (Davidson and Fullerton), that these functions, together with the requirements of lactation, demand 900 milligrammes of iron.

This amounts to a need for a daily positive balance of 2 milligrammes of iron, which is not a great amount; and it is probable that hypochromic anaemia does not develop in pregnant women as a result of simple iron-deficiency directly due to uncomplicated pregnancy, if the state of health was previously good (see below). 
The idea of a physiological anaemia of pregnancy is not based on accurate haematological observation. Normally, there is no anaemia, at least in the sense of a diminished production of red corpuscles and haemoglobin; any decrease, in normal pregnancy, is the result of hydraemia.

The blood volume is increased very considerably during pregnancy, beginning about the third month (Thomson et al.); and it is estimated that, at term, the increase of the blood volume is 23 per cent, and that of the plasma 25 per cent (Dieckmann and Wegner). This may be assumed to be the reason that loss of blood is so well tolerated by pregnant and parturient women.

The blood volume has returned to normal within a few weeks of delivery.

The leucocyte count increases steadily throughout pregnancy, mainly by addition to the number of neutrophiles, most of which are normal mature polymorphs; but, in the last weeks, there is some increase of less mature forms, with simple indented (i.e. unlobed) nuclei: the staff cells of Schilling. The emergence of these cells can be taken as an indication that the marrow is meeting the demand for white cells with some difficulty.

During labour, the number of leucocytes rises steeply, often reaching 35,000 per c.mm., but this is a transient state; and, in the absence of complications, the number of white cells has returned to normal by the end of the puerperium.

The sedimentation rate is increased from the third month onwards; and this may cause considerable perturbation if it is not recognised as a normal phenomenon. Leucocytosis, due to increase of neutrophiles, some of which are somewhat immature (shift to the left of the Arneth and Schilling counts), together with the increased sedimentation rate are liable to mislead the unwary into supposing that an infection is present when, as a matter of fact, the changes are entirely due to the pregnancy.

A great deal more work needs to be done on the normal changes in the white cells during pregnancy, because, at present, our knowledge is too incomplete to allow of sound inferences being drawn in many cases of intercurrent infection.

For example, such an acute abdominal emergency as a ruptured tubal gestation may simulate an acute infective state, both clinically and haematologically, because there is great increase in the number of leucocytes (even up to 25,000 per c.mm.).

If surgeons continue to practice the bad habit of asking for a total leucocyte count, without asking for a differential count of the leucocytes (including at least the Schilling haemogram), there will continue to be gross errors of interpretation of blood pictures.

In infective conditions of any considerable degree of severity there will be not only increase of neutrophiles, with some more or less immature forms, but also some cells showing degenerative changes, such as vacuolation of the cytoplasm, clumping of the granules, and nuclear pyknosis. On the other hand, the leucocytosis of normal pregnancy is produced entirely by cells which are normal in structure. It is clear, therefore, that, if blood examination is to give any information of real value in conditions suspected of being infective in origin, a most detailed examination of the white cells is necessary.

The need for care is further shown by the fact that leucocytosis occurs after any haemorrhage, but, if the bleeding is on the surface, the increase of white cells is only slight, whereas, if it is in contact with the peritoneum, dura, pleura, or a joint surface, the increase is very much greater, even in the absence of infection.

\section{ANAEMIA IN PREGNANCY}

As mentioned above, it is probably true to say that no anaemia is truly physiological, but anaemia of such slight degree that it does not interfere with health is so common in pregnant women, that it is often considered to be a normal concomitant of the condition.

This mild anaemia presents a colour index below $I \cdot 0$, while the average diameter of the red corpuscles is within normal limits: it is thus a normocytic hypochromic anaemia.

Kühnel stated that a fall of red corpuscles to $3,500,000$ per c.mm. was within "physiological" limits, but there are probably very few physicians who would agree that such a state should be regarded so lightly. Hypochromic anaemia of such severity calls for immediate treatment with iron, especially as, in untreated cases, there is a tendency for both the red corpuscles and haemoglobin to fall still further from the thirty-second week onwards.

The part played by iron-deficiency in the genesis of this kind of anaemia is shown by the prompt improvement in the blood-picture when adequate doses of iron are given. Also, in untreated cases, the red corpuscles rise rapidly to normal numbers during the puerperium, but 
the haemoglobin lags behind; that is to say, the colour index falls still further. Unless iron is given, the haemoglobin may remain low for weeks or months; but, if iron is exhibited, there is rapid increase. It seems clear that the body was short of the iron needed for the formation of the respiratory pigment, although the substances needed for formation of corpuscles were present in adequate amount.

It is probable that at least two factors play a part in the genesis of this form of anaemia, viz., initially defective stores of iron in the body (sometimes for dietetic reasons), and inadequate absorption from the stomach on account of the hypochlorhydria that develops in so many pregnant women (see below).

\section{Hypochromic Anaemia of Pregnancy.}

Strictly, there is no such condition as hypochromic anaemia of pregnancy, because there is no difference between it and similar anaemia arising in the non-gravid: pregnancy is simply the factor that precipitates or aggravates the iron deficiency which is the cause of the anaemia. In other words, there is no real difference of kind, but only of intensity, between this form of anaemia and the "physiological" anaemia discussed above.

The disease is commonest in multiparae especially of the poorer classes; and it is reasonable to assume that these are women whose blood has never returned to normal after their previous pregnancies, and that they have never acquired properly stored iron depots. Thus, the new pregnancy is started with an inadequate store of iron, and even with some degree of hypochromic anaemia. Indeed, a history of anaemia prior to the pregnancy can often be obtained (Fullerton; Strauss and Castle).

In other cases, anaemia does not manifest itself until about the middle of pregnancy; that is, at a time when an initially small store of iron is becoming further depleted by the demands of the foetus.

Not only is there the factor of inadequate stores of iron in the maternal tissues, but there is also that of defective absorption from the gastro-intestinal tract, which depends upon bypochlorhydria or achlorhydria, which is so common in pregnancy. Thus, Strauss and Castle, ing a series of 29 cases of chronic hypochromic anaemia in pregnancy, found normal gastric acidity in only two patients, while achlorhydria, which persisted after delivery, was present in $17 . \oplus$ Of course, infection and toxaemia may also play a part: not everyone with achlorhydria suffers? from iron-deficiency anaemia.

It may thus be accepted that the hypochromic anaemia of pregnancy is not a disease sui generis, but is identical with the ordinary form of the malady as seen in non-gravid persons. All that has happened is that the increased demand for iron, both by the physiologically hydraemic mother and by the foetus, cannot be fully met from the stores in the body, which may start in a depleted state, and which are not replenished, either because of.poor absorption, defective diet, or sepsis, or all these factors.

\section{Megalocytic Hyperchromic Anaemia of Pregnancy.}

The term "pernicious anaemia of pregnancy" is so vague, that it is better to relegate it to an historical museum and to replace it by the descriptive name, megalocytic hyperchromic anaemia, which has the advantage of indicating the essential features of the blood picture.

Much of the confusion is due to the older writers, who used the adjective "pernicious" to mean nothing more than severe and dangerous; but, in modern terminology, by pernicious anaemia is meant a malady due to deficiency of haemopoietic factor. If this definition is accepted, there can be no dispute that pernicious anaemia does sometimes develop in association with pregnancy; but this state must be distinguished from a pregnancy which is only an incident in the course of ordinary cryptogenetic pernicious anaemia. This is, of course, an uncommon occurrence, because, as is generally known, pernicious anaemia is a disease of middle and later life.

Incidentally, there is an interesting point in connection with pregnancy in cases of cryptogenetic pernicious anaemia, because this is, not uncommonly, a familial malady; and there thus arises the question whether the child is likely to develop the disease. It may be said that there is no sound reason for advocating eugenic abortion in such cases, because the chances of pernicious anaemia developing in the child are not great; and, even if it does occur, it is unlikely to do so until middle-age, when much of its life-work will have been done. Then again, treatment is so efficacious that its well-being can be preserved for many years. 
The pernicious anaemia of pregnancy is something very different from cryptogenetic pernicious anaemia, although some of the symptoms and the characters of the blood-picture are the same.

In these cases, about the middle of pregnancy, there develop gastro-intestinal disturbances, pallor and soreness of the tongue (but this is not invariable). A sub-icteric tinge, with positive indirect van den Bergh reaction (slight haemolytic jaundice) is common, and resembles the slight icterus of pernicious anaemia.

Achlorhydria, which is practically constant in cryptogenetic pernicious anaemia, is not so in the pernicious anaemia of pregnancy; indeed, it is less common than in pregnant women who are suffering from chronic hypochromic anaemia.

Another difference is that damage to the nervous system does not develop; and it is clear that the condition is not identical with the idiopathic malady.

Again, when recovery has taken place, spontaneous relapses do not occur, even if all treatment is stopped. Another attack may, or may not, develop in a subsequent pregnancy (Whitby), but, apart from that possibility, there appears to be no more chance of a woman who has suffered from pernicious anaemia of pregnancy developing the idiopathic disease than of anyone else doing so.

The blood-picture has the general characters of idiopathic pernicious anaemia: megalocytosis, hyperchromia, widened Price Jones curve, leucopenia, and relative lymphocytosis Even so, the intensity of the megalocytosis is less than in the cryptogenetic disease, and it is very rare for the anaemia to become as severe.

The bone-marrow, as examined in films made from material obtained by sternal puncture, shows the presence of many megaloblasts identical with those of pernicious anaemia (Davidson et al.), but their number is not as great, and there is, consequently, a good deal more evidence of normoblastic activity than in ordinary untreated pernicious anaemia.

Treatment, during the pregnancy, consists of injections of liyer extract; but the response is often less prompt and less satisfactory than in ordinary pernicious anaemia. In severe cases, the patient may need one or more blood transfusions to tide her over the pregnancy and delivery, but the liver injections must be continued during the whole time.

It is essential not to wait until the patient is very severely anaemic before resorting to transfusion: the fatal cases are usually those in which there has been undue delay in giving a transfusion, because the physician has expected as good a response to liver treatment as he would get in the ordinary form of the disease.

It has often been stated that the pernicious anaemia of pregnancy is cured spontaneously after delivery; but this is far from being invariable. Treatment should be continued with as much energy after parturition as before, until the blood-picture has been restored to normal. If one waits for the spontaneous recovery that has been promised by some writers, one will find that some women become even more anaemic after delivery, and may even need more transfusions.

The chances of death after delivery in a well-treated case are very small, but, if a spontaneous improvement is waited for, there is a far from negligible chance of the anaemia getting out of hand and proving fatal.

The reason for the relatively poor response to liver treatment is not known; but there is one factor in treating these patients that needs care and attention if restoration to complete normality is to be attained.

As pointed out above, iron-deficiency is common in pregnant women, especially in multiparae of the poorer classes; and, although this does not predispose to the development of the pernicious anaemia of pregnancy, it does very considerably complicate the blood picture and the problem of treatment. Thus, in pernicious anaemia, there are considerable stocks of iron in the body, partly because it is not used up and partly on account of haemolysis. As a rule, the amount of iron is sufficient to supply haemoglobin to all the corpuscles that are formed in response to liver treatment; but, if the stores of iron are initially low, there may be insufficient to do so. Then we have a so-called dimorphic anaemia, in which there is (or has been) deficiency ot haemopoietic factor and also of iron. In the later stages of liver treatment, the red corpuscles will continue to rise, but the haemoglobin lags behind, and the colour index falls.

No harm is done by administering iron in the later stages of treatment in all cases, but, for scientific accuracy, it is better to have fairly frequent blood counts, so that the appropriate time for giving iron can be found. 
The treatment of the pernicious anaemia of pregnancy is so satisfactory, that it can be said that the disease is not an indication for therapeutic abortion. Indeed, as the anaemia may become more severe after normal labour, it may also do so after abortion; and there is thus no benefit to the patient in causing her to lose her child.

The genesis of the disease is far from being clear. Of course, there is a deficiency of haemopoietic factor: hence, the megalocytic hyperchromic anaemia; but this is not a complete explanation. In idiopathic pernicious anaemia, the deficiency of haemopoietic factor: is the certain cause of the morbid condition; and administration of the missing material results in rapid return to normal.

The pernicious anaemia of pregnancy does not respond so promptly and certainly to liver treatment; and it must be assumed that there is some unknown factor in addition to deficiency of intrinsic factor in the stomach. Certainly, in the great majority of cases, there is no dietetic deficiency; that is to say, the diet before the pregnancy is not at fault.

\section{Tropical Megalocytic Anaemia.}

This condition, which has been so carefully studied by Wills and her colleagues in India, and by Fairley in Macedonia, presents a blood picture similar to that of pernicious anaemia, viz. megalocytosis and hyperchromia; but, unlike that disease, the origin of the malady is mainly dietetic: the deficiency is one of extrinsic factor.

In India, for example, the dietary may be gravely restricted for economic or religious reasons; and the deficiency of extrinsic factor prevents the normal interaction with intrinsic factor for the elaboration of the haemopoietic principle. As would be expected, the diet is not, as a rule, poor in only one particular; and, for this reason, dimorphic anaemia is common, i.e. an anaemia due to deficiency of haemopoietic principle, associated with one, often temporarily overshadowed by the first, resulting from inadequate amounts of iron in the food.

At first, the anaemia is megalocytic and usually hyperchromic; but, after energetic treatment, the iron-deficiency manifests itself, and the colour index falls greatly.

Clearly, treatment must be based on our knowledge of the aetiology of the condition: ex- 8 trinsic factor must be supplied in order to combat the hyperchromic anaemia, i.e. to cause the mode of erythrocytopoiesis in the marrow to become normoblastic; and iron must also be supplied.co

The highly purified liver extracts that are so efficacious in pernicious anaemia are less so in this form of disease; but the much cruder extracts are admirable. Indeed, the residue left when purified liver extracts are being made is very efficacious (Alsted).

Of course, the diet must also be amended so that there is an ample supply of extrinsic factor; and oral treatment with substances containing a great deal of this substance is perfectly satisfactory, while being much cheaper than liver treatment. Marmite contains a great deal of extrinsic factor; and its administration in large doses rapidly relieves the "pernicious" anaemia.

Iron should be given in all cases, because it is so probable that the manifold defects of the diet include this one also.

This form of megalocytic anaemia is not common in temperate climates: on the whole, the standard of living is too high to permit of it; but, in India and also in Macedonia, the malady is common and is a grave cause of maternal morbidity and even mortality.

The importance of a properly balanced diet is shown by the fact that, if a remission is induced by liver injections or by Marmite, together with iron, no relapse occurs if the patient is supplied with an ordinary mixed diet (Groen and Snapper).

\section{PURPURA}

A few small purpuric spots may develop in any pregnant woman; but, in cases of hyperemesis and of eclampsia, moderately severe purpura is not uncommon. This is not associated with reduction in the number of platelets, but appears to be allied to such anaphylactoid purpuric states as Schönlein's disease and Henoch's purpura.

Such intercurrent purpura does not seem to affect the prognosis at all; but the co-existence of pregnancy and purpura haemorrhagica (thrombocytopenic purpura) is a grave condition. Thus, in a series of 44 cases of this combination, Rushmore records 26 maternal deaths; only I5 infants survived; and 7 instances of purpura in the foetus were observed. Wintrobe has mentioned thrombocytopenia in the baby of a woman suffering from thrombocytopenic purpura, although there was no excessive bleeding. 
As a rule, the mother's blood platelets fall lower as the pregnancy progresses; and it seems probable that early therapeutic abortion might save most of the maternal lives. If abortion is not followed by rapid improvement, transfusions can be given, and even splenectomy can be performed with much less danger on a woman who has been aborted in the early stages of pregnancy than in a parturient one.

If abortion is refused, the condition is by no means hopeless, but treatment must be continuous and carefully controlled by frequent platelet counts. Thus, intramuscular injections of small amounts of blood tend to keep the platelets from falling, as do other forms of mild protein shock. It has also been claimed that injection of fairly crude liver extracts have a beneficial effect; but this is by no means certain.

Whatever treatment is adopted, means must always be at hand for transfusion at short notice, and even for splenectomy.

In order to avoid excessive bleeding during labour, every possible step should be taken to raise the number of platelets. Injection of Io minims of adrenalin cause a rapid, but transient, thrombocytosis, but can be given several times during labour to prevent excessive loss of blood. But whatever is done, transfusion may become imperative; and no time must be lost.

\section{HAEMOLYTIC CONDITIONS DURING PREGNANCY}

There are no forms of essentially haemolytic anaemia peculiar to pregnancy, but such a state may be present before pregnancy or may develop during it.

A woman suffering from acholuric family jaundice does not appear to be more likely to come to harm during pregnancy than at any other time; but, of course, an exacerbation, a haemolytic crisis, may develop just as in the non-gravid. The problem is then a difficult one, because abortion will not stop the haemolytic process, and splenectomy is extremely dangerous during acute exacerbations of the disease.

Frightening as the condition may be, with its increasing jaundice and anaemia, it is probably best to do nothing except keep the patient in bed until recovery commences: death very rarely occurs in untreated cases. Transfusion is very likely to evoke extreme reactions, which may themselves prove fatal; and it is wise not to use this method of treatment unless it seems as if the anaemia itself is likely to prove fatal.

Pregnancy may occur in sufferers from the rare syndrome of nocturnal haemoglobinuria, but, strangely enough, the patient may improve during that time, the haemolysis becoming minimal (Hamburger and Bernstein). There is, thus, no reason for terminating the pregnancy; but, if the malady persists or becomes aggravated, transfusions may be tried. These usually cause severe reactions, but, if not fatal, are often followed by considerable improvement.

The rather obscure disease, known as Lederer's anaemia, seems to occur more frequently in pregnant women than in others (Witts).

The cause of the disease is unknown, but the clinical features are those of an acute infection: sudden onset of fever, vomiting, headache, and malaise, with rapidly progressive pallor.

The blood shows anaemia of haemolytic type, i.e. little change in the colour index, but great reticulocytosis. The white cells are greatly increased (again, resembling an infective condition), and the indirect van den Bergh reaction is positive.

The patient is extremely ill, but, even so, transfusion, which may need to be repeated once or twice, brings the haemolytic process to a prompt end. Specially careful matching of the bloods is essential, because there is a great tendency to autoagglutination.

Two things are clear: evacuation of the uterus does not do any good, and prompt transfusion, repeated as may be necessary, will obviate any grave danger to life.

Puerperal infection is a subject that lies outside our present purview; but it will be well to mention that severe anaemia, partly due to haemolysis, may result from streptococcal and staphylococcal infection. The treatment is, of course, on general lines, but transfusion should never be long delayed, both in order to make good the anaemia and, at the same time, to supply the patient with complement, which may be scanty in her serum.

\section{THE LEUKAEMIAS}

Leukaemia is no more likely to develop during pregnancy than at any other time, but a leukaemic woman may become pregnant.

If the leukaemia is of the acute type, spontaneous abortion is almost certain to occur; but, 
in the chronic types, the pregnancy usually proceeds normally. There is no reason for terminating the gestation, because the children of leukaemic mothers do not appear to be affected by the disease (Chiari and Dantwitz). There is some danger of excessive bleeding during delivery, but this risk is at least as great after therapeutic abortion. Prompt transfusion will, almost certainly, prevent a fatal result.

The prognosis of the mother's disease does not appear to be affected by pregnancy, except that X-ray treatment is inadvisable during pregnancy, and the malady may, therefore, progress unchecked. On the other hand, arsenic, administered by mouth, will usually keep the disease within bounds until after delivery, when, if necessary, irradiation can be started again.

\title{
CONCLUSION
}

It may be said that the anaemias that are likely to develop during pregnancy do not, as a rule, greatly endanger life; but, unless adequately treated, may become a potent factor in the development of chronic ill-health. Only those who do many blood examinations on women, who allege that they have not felt well since the baby was born, will realise how often a moderate degree of hypochromic anaemia is the easily removable cause. It is, perhaps, because most of the haemic disorders of pregnancy are not spectacular in their clinical picture or in their immediate effects, that has led to their neglect, much to the detriment of women's health.

\section{BIBLIOGRAPHY}

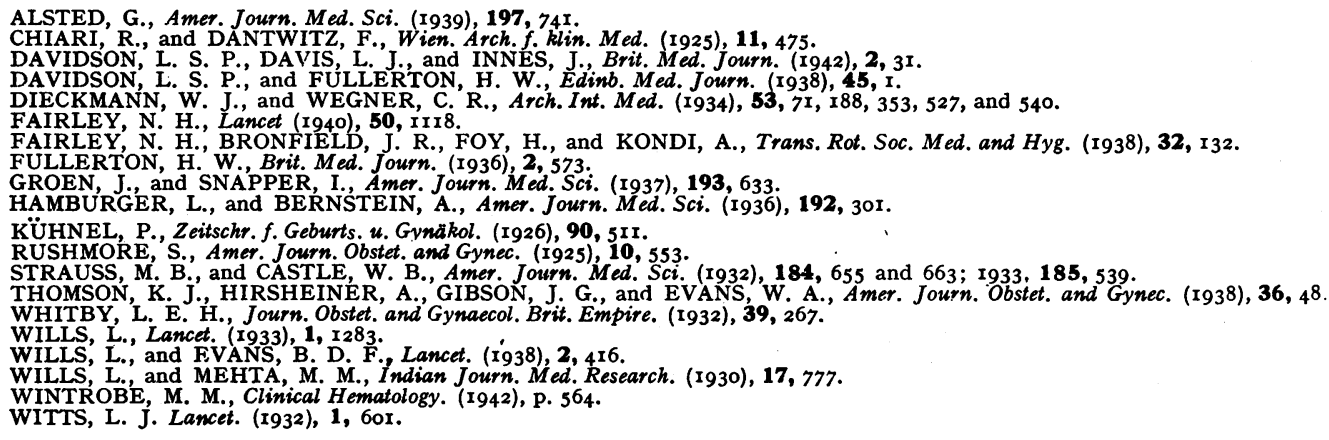

\section{HEART DISEASE IN PREGNANCY}

\author{
By A. MORGAN JONES, M.Sc., M.B., M.R.C.P. \\ (From the Cardiac Pregnancy Clinic, Manchester Royal Infirmary)
}

When a pregnant woman has heart disease the physician's problem is threefold: first, to determine the nature of the cardiac lesion; next, to assess the patient's fitness for pregnancy, and finally, to undertake any necessary supervision and treatment of the cardiac condition. It is therefore convenient to discuss the problem from the three aspects of diagnosis, assessment and management.

\section{DIAGNOSIS}

If heart disease has not been previously recognised, it is often suspected during pregnancy if the patient complains of breathlessness, swelling of the ankles, palpitation or pain in the region of the heart, or when routine antenatal examination reveals irregularity of the pulse, or a cardiac murmur. Such symptoms and signs are not invariably due to heart disease, and in 